\title{
Edge-spin accumulation in semiconductor two-dimensional hole gases
}

\author{
K. Nomura, ${ }^{1}$ J. Wunderlich, ${ }^{2}$ Jairo Sinova, ${ }^{3}$ B. Kaestner, ${ }^{2,4}$ A. H. MacDonald, ${ }^{1}$ and T. Jungwirth ${ }^{5,6}$ \\ ${ }^{1}$ Department of Physics, University of Texas at Austin, Austin, Texas 78712-1081, USA \\ ${ }^{2}$ Hitachi Cambridge Laboratory, Cambridge CB3 OHE, United Kingdom \\ ${ }^{3}$ Department of Physics, Texas A\&M University, College Station, Texas 77843-4242, USA \\ ${ }^{4}$ National Physical Laboratory, Teddington T11 OLW, United Kingdom \\ 5Institute of Physics ASCR, Cukrovarnická 10, 16253 Praha 6, Czech Republic \\ ${ }^{6}$ School of Physics and Astronomy, University of Nottingham, University Park, Nottingham NG7 2RD, United Kingdom
}

(Received 22 August 2005; revised manuscript received 28 October 2005; published 21 December 2005)

\begin{abstract}
The controlled generation of localized spin densities is a key enabler of semiconductor spintronics In this work, we study spin Hall effect induced edge-spin accumulation in a two-dimensional hole gas with strong spin orbit interactions. We argue that it is an intrinsic property, in the sense that it is independent of the strength of disorder scattering. We show numerically that the spin polarization near the edge induced by this mechanism can be large, and that it becomes larger and more strongly localized as the spin-orbit coupling strength increases, and is independent of the width of the conducting strip once this exceeds the elastic scattering mean-free-path. Our experiments in two-dimensional hole gas microdevices confirm this remarkable spin Hall effect phenomenology. Achieving comparable levels of spin polarization by external magnetic fields would require laboratory equipment whose physical dimensions and operating electrical currents are a million times larger than those of our spin Hall effect devices.
\end{abstract}

DOI: $10.1103 /$ PhysRevB.72.245330

PACS number(s): 75.50.Pp, 85.75.Mm

\section{INTRODUCTION}

Finite spin densities in semiconductors have traditionally ${ }^{1}$ been generated by external magnetic fields, by circularly polarized light sources, or by spin injection from ferromagnets. Recently there has been considerable interest ${ }^{2}$ in an alternate strategy in which edge-spin densities are generated electrically via the spin Hall effect (SHE), 3,4 i.e., in a planar device by the current of spins oriented perpendicular to the plane that is generated by and flows perpendicular to an electric field. The SHE has traditionally been thought of as a consequence of spin-dependent chirality in impurity scattering that occurs in systems with spin-orbit (SO) coupling. ${ }^{5,6}$ Recently it has been recognized that the SHE also has an intrinsic contribution due to SO coupling in a perfect crystal. ${ }^{7,8}$ The theoretical works ${ }^{6-8}$ and the subsequent experimental discovery $^{3,4}$ of the SHE in both strong and weak SO interaction regimes have generated substantial interest in both the origins of this unfamiliar transport phenomenon and its potential for low power consumption electronic devices. ${ }^{3,7}$ The role of disorder in the spin-Hall conductivity is more subtle than in more familiar transport coefficients. Early theoretical work reflected a variety of strongly polarized views, all the way from a picture with large dissipationless transverse spin currents $^{7,8}$ in which disorder plays no role, to demonstrations that the effect is completely eliminated by infinitesimally weak disorder. ${ }^{9-12}$ The theoretical picture is now becoming more clear, if more complex. It is now established that many of the theoretical controversies have arisen because what is seemingly the simplest possible model system, a twodimensional electron gas with Rashba SO coupling, ${ }^{13}$ turns out to have anomalous properties. Although the SHE is absent in this model, it is present and robust against disorder ${ }^{14-17}$ in generic systems and in all other models that have been specifically studied.
Another challenging issue in the SHE theory concerns the definition of the spin current in SO coupled systems; since spin is not conserved, the merit of a particular definition has to be decided on the basis of its utility for the evaluation of observable properties. The fact that different definitions have been proposed which yield spin Hall currents that differ even by a sign for some models emphasizes the importance of this point. ${ }^{18-20}$ The most important observable associated with the SHE is the edge spin accumulations to which it gives rise; indeed experiments that discovered the $\mathrm{SHE}^{3,4}$ did so by measuring edge spin accumulations. These measurements have partly steered the focus of theoretical studies from the experimentally ambiguous spin Hall currents to spin Hall accumulations. ${ }^{21-24}$ The opportunity to directly compare theoretical and experimental values of a SHE related physical quantity has been appealing, yet physicists have entered this territory armed with no analytical theory that is able to predict the sign, magnitude, and spatial extent of the SHE spin accumulation, at least in the most interesting case of strong SO interactions. Particularly intriguing in this context is the dual role of the SO coupling which generates the spinpolarization of carriers while, at the same time, causing spin decoherence upon scattering off channel edges or off impurities. In the present paper we compare a numerical study and experimental results of the SHE spin accumulation in twodimensional hole gases (2DHGs). The systems we study are in the diffusive transport regime in which the sample dimensions are large compared to scattering mean-free-path.

\section{THEORY}

First we discuss our theoretical results. The calculations are based on the following model Hamiltonian ${ }^{25,26}$ of strongly confined $2 \mathrm{DHG}$ at semiconductor heterojunction with structural inversion asymmetry 


$$
H_{R}=\left(\begin{array}{rr}
\hbar^{2} k^{2} / 2 m & i \lambda k_{-}^{3} \\
-i \lambda k_{+}^{3} & \hbar^{2} k^{2} / 2 m
\end{array}\right)
$$

where $m$ is the carrier effective mass and the momenta $k$ $=\left(k_{x}^{2}+k_{y}^{2}\right)^{1 / 2}, k_{ \pm}=k_{x} \pm i k_{y}$, and $\lambda$ is the SO coupling constant due to structure inversion asymmetry across the quantum well grown along the $z$ axis. The 2DHG strip is defined by hard wall barriers separated by a distance $L_{x}=L$ in the transverse $x$ direction and by a longitudinal length $L_{y}=L$, and periodic boundary condition in the $y$ direction. Disorder is modeled assuming uncorrelated, short-range spinindependent scatterers, i.e., $V(\mathbf{r})=\sum_{I=1}^{N_{i}} V \delta\left(\mathbf{r}-\mathbf{R}_{I}\right)$. This "white-noise" disorder potential satisfies $\left\langle V(\mathbf{q}) V\left(\mathbf{q}^{\prime}\right)^{*}\right\rangle$ $=N_{i} V^{2} \delta\left(\mathbf{q}-\mathbf{q}^{\prime}\right)$ where $V(\mathbf{q})$ is the Fourier component of $V(\mathbf{r})$ and $N_{i}=n_{i} L^{2}$ is the number of impurities; $n_{i} \approx n$ where $n$ is the 2DHG density. The strength of disorder can be characterized by the corresponding Born approximation scattering rate, $\hbar / \tau=2 \pi n_{i} V^{2} \rho_{F}$, where $\rho_{F}$ is the $2 \mathrm{D}$ density of states in the absence of SO coupling. In the following we relate all energies to the Fermi energy, $E_{F}$, of the 2DHG in the absence of SO coupling and disorder, and all lengths to the corresponding inverse Fermi wave vector $k_{F}^{-1}$. In our studies, $E_{F}$ is always the largest relevant energy scale.

The SHE spin-accumulation is generated near the edges of the system by a charge current in the $y$ direction. The linear response theory predicts an induced spin density given by the Kubo formula

$$
\begin{aligned}
S_{z}(x)= & -i \hbar E_{y} \int_{0}^{L_{y}} \frac{d y}{L_{y}} \sum_{\alpha, \alpha^{\prime}} \frac{f\left(E_{\alpha}\right)-f\left(E_{\alpha^{\prime}}\right)}{E_{\alpha}-E_{\alpha^{\prime}}} \\
& \times \frac{\left\langle\alpha\left|s^{z}(x, y)\right| \alpha^{\prime}\right\rangle\left\langle\alpha^{\prime}\left|j_{y}\right| \alpha\right\rangle}{E_{\alpha}-E_{\alpha^{\prime}}+i \eta},
\end{aligned}
$$

where $s^{z}(x, y)$ is the $z$ component of the local spin density operator (we assume spin $3 \hbar / 2$ for the heavy holes), $j_{y}$ $=e \partial H / \partial \hbar k_{y}$ is the longitudinal electrical current operator for holes, and $E_{\alpha}$ and $|\alpha\rangle$ are the eigenenergies and eigenstates of the full Hamiltonian $H$ which includes the SO coupling term (1), the disorder potential, and the hard-wall confining potential. $\eta$ must be chosen to be small compared to all intensive energy scales, such as $E_{F}, \hbar / \tau$, and the characteristic SO coupling strength $\Delta_{s o}=2 \lambda k_{F}^{3}$, but larger than the finite system level spacing. A detailed discussion of subtleties related to the proper choice of $\eta$ can be found in Ref. 17 .

Typical results of the calculated spatial profile of spindensity induced by a longitudinal electric field $E_{y}$ are shown in Fig. 1(a). For comparison we show in Fig. 1(b) the transverse spin current, $j_{x}^{z} \equiv\left\{s_{z}, v_{x}\right\} / 2$, also obtained using the linear response theory. ${ }^{8,17,26}$ In both panels, $\Delta_{s o} / E_{F}$ $=0.4,(\hbar / \tau) / E_{F}=0.3$, and the diffusive regime is obtained by assuming system size $L=50\left[k_{F}^{-1}\right]$ which is larger than the mean free path, $l=6\left[k_{F}^{-1}\right]$. The $z$ component of the induced spin polarization has maxima near the strip edges, while the spin current has a maximum in the middle of the sample and decays toward the edges. We note that the peak value of the spin current divided by the applied electric field is comparable to the spin Hall conductivity calculated in a previous

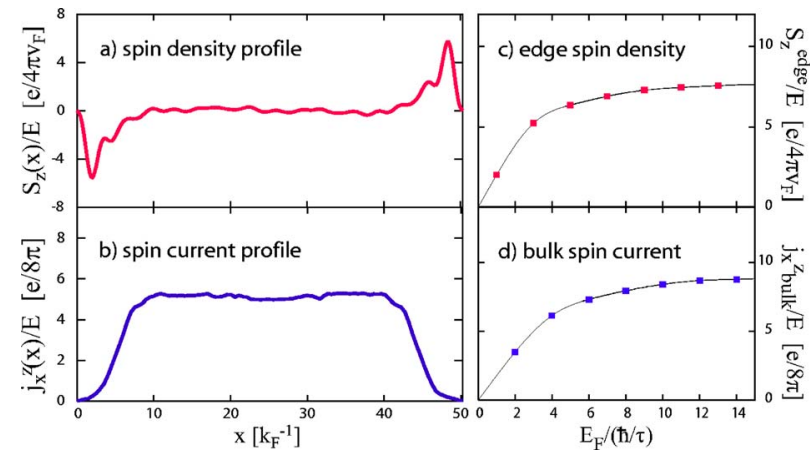

FIG. 1. (Color online) Spin Hall effect in a 2DHG. Spatial profile of the $z$ component of spin (a), and of the spin current (b) across the channel width ( $x$ direction). Disorder dependence of the accumulated edge-spin density (c), and of bulk spin current (d).

study with periodic boundary conditions rather than hard walls in the $x$ direction, and to analytical calculations which account for disorder by including finite quasiparticle lifetime broadening. ${ }^{17,26}$

In Figs. 1(c) and 1(d) we illustrate the disorder dependence of the peak value of the edge-spin density $S_{\text {edge }}^{z}$, and of the transverse spin current in the middle of the strip $j_{x, b u l k}^{z}$. With increasing disorder strength, both $S_{\text {edge }}^{z}$ and $j_{x, \text { bulk }}^{z}$ decrease gradually and vanish in the strong scattering limit $\hbar / \tau \gg \Delta_{s o}$. The striking similarity of the dependences on disorder strength of the two quantities led us to a numerical observation that

$$
v_{F} S_{\text {edge }}^{z} \sim j_{\text {bulk }}^{z},
$$

where $v_{F}$ is the hole Fermi velocity. Introducing the characteristic SO coupling length $L_{s o}=v_{F} \pi \hbar / \Delta_{s o}$ and time $t_{s o}$ $=\hbar \pi / \Delta_{s o}$, we can rewrite Eq. (3) as

$$
L_{s o} S_{\text {edge }}^{z} \sim t_{\text {so }} j_{\text {bulk }}^{z} \text {. }
$$

Here we emphasize that both $L_{s o}$ and $t_{s o}$ have an intrinsic, disorder independent origin. Equation (4) suggests that the spin polarization accumulated in the region of size $L_{s o}, L_{s o} S_{\text {edge }}^{z}$, is proportional to the amount of incoming spin current from the middle of the sample in a time scale $t_{s o}$.

To further examine the validity of this ansatz (4) we plot in Figs. 2(a) and 2(b) the dependence of the spin polarization profile on $\Delta_{s o}$ and on $\hbar / \tau$. As seen in Fig. 2(a), the spin density builds up more gradually from the center towards the edge of the strip for weaker SO coupling. Figure 2(b) demonstrates explicitly that the envelope of the spin polarization curve changes only weakly with disorder. Calculations presented in Fig. 2(c) further demonstrate that the edge spin accumulation properties do not change once the system size exceeds the SO length $L_{s o}$.

Our findings on the disorder dependence of spinaccumulation differ from expectations based on analogies to other regimes. In the case of strong disorder the accumulation time scale is the spin-relaxation time ${ }^{5} \tau_{s} \sim \Delta_{s o}^{-2} \tau^{-1}$ while the accumulation length scale is the spin-diffusion length $l_{s}$ $=\sqrt{D \tau_{s}}$ where $D$ is the diffusion coefficient. For a given spin Hall current, analogies with this regime predict ${ }^{7,19,20}$ accu- 

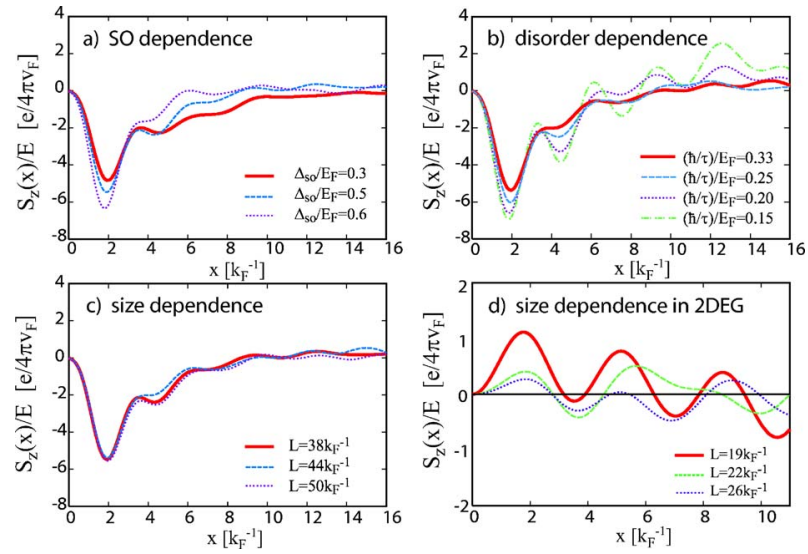

FIG. 2. (Color online) Spatial profile of the $z$ component of accumulated spin density as a function of spin-orbit coupling for $(\hbar / \tau) / E_{F}=0.3$ and $L=44 k_{F}^{-1}$ (a), the disorder strength for $\Delta_{s o} / E_{F}$ $=0.4$ and $L=44 k_{F}^{-1}(\mathrm{~b})$, and of the system size for $(\hbar / \tau) / E_{F}=0.3$ and $\Delta_{s o} / E_{F}=0.4$ (c). System size dependence in a $2 \mathrm{DEG}$ for $(\hbar / \tau) / E_{F}=0.15$ and $\Delta_{s o} / E_{F}=0.4(\mathrm{~d})$.

mulation on the spin diffusion length $l_{s}$ or the spinprecession length scale $L_{s o},{ }^{27}$ which corresponds to $l_{s}$ in the strong disorder limit, ${ }^{5}$ and predict that the magnitude is proportional to $\tau_{s} \propto \tau^{-1}$. The applicability of this analogy in the strong SO scattering limit is not established theoretically, however, and our numerical results suggest that it is doubtful. Consistency is not achieved by simply replacing the weak SO interaction expression for the spin relaxation time by the strong SO interaction result that $\tau_{s} \sim \tau$, which would imply that the spin accumulation is proportional to $\tau^{7,19,20}$

We can understand why the Kubo formula predicts that the induced spin-density is weakly dependent on scattering rate in the diffusive limit by considering the matrix elements of the spin-density and current operators in Eq. (2). Because the paramagnetic system is even under time-reversal and the spin-density is odd, it follows that its static linear response originates from the odd dissipative term in the Kubo formula

$$
\begin{aligned}
S_{z}(x)= & -\pi \hbar E_{y} \int_{0}^{L_{y}} \frac{d y}{L_{y}} \sum_{\alpha, \alpha^{\prime}} \frac{\partial f\left(E_{\alpha}\right)}{\partial E_{\alpha}}\left\langle\alpha\left|s^{z}(x, y)\right| \alpha^{\prime}\right\rangle \\
& \times\left\langle\alpha^{\prime}\left|j_{y}\right| \alpha\right\rangle \delta_{\eta}\left(E_{\alpha}-E_{\alpha^{\prime}}\right),
\end{aligned}
$$

where $\delta_{\eta}(E) \equiv \eta / \pi\left[E^{2}+\eta^{2}\right]$. Typical properties of these matrix elements are illustrated in Fig. 3(a) and 3(b) where by "ME" we denote the product of the spin and current matrix elements in Eq. (5). As in all transport properties, current operator matrix elements in the absence of disorder play a key role. For $2 \mathrm{DHG}$ strips, the component of momentum along the strips is a good quantum number whereas the transverse momentum is replaced by discrete standing wave or one-dimensinal (1D) subband indices. Both spin and charge operators have matrix elements that are diagonal in the 1D wave vector and in the subband index. It follows that the induced spin-density that we calculate is (at $T=0$ ) proportional to $\eta^{-1}$ in the absence of disorder, diverging when the $\eta \rightarrow 0$ limit is taken. The unsurprising disorder, which limits the lifetime of these $1 \mathrm{D}$ band states, must be considered to
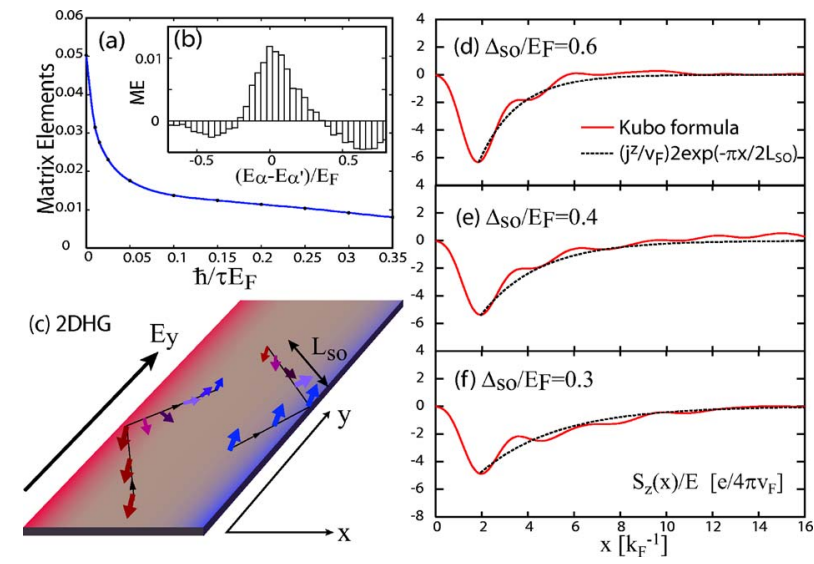

FIG. 3. (Color online) Matrix elements that appear in Eq. (5) as a function of the scattering rate at $E_{\alpha}-E_{\alpha^{\prime}}=0$ (a), and as a function of the energy difference at $(\hbar / \tau) / E_{F}=0.14$ (b). $\Delta_{s o} / E_{F}=0.3$ and $L$ $=44 k_{F}^{-1}$ in both panels. (c) Schematic of the spin Hall accumulation in the strong SO coupling regime. Spatial profile of $z$ component of spin density for (d), $\Delta_{s o} / E_{F}=0.6,0.4$ (e), and 0.3 (f) at $(\hbar / \tau) / E_{F}$ $=0.3$.

obtain a finite result. In the limit of very weak disorder, the lifetime broadening by $(\hbar / \tau)$ of the band states is smaller than the subband separation, and it follows from Eq. (5) that the induced spin-density will be proportional to $\tau{ }^{28,29}$ This is the limit in which the 1D subbands contribute independently to the induced spin density and can be treated as separate bands in a Boltzmann transport theory.

The 1D band limit applies only when $\hbar / \tau$ is smaller than the subband splitting, i.e., when $\hbar / \tau<\hbar v_{F} / L_{x}$ and therefore when the mean free path $l=v_{F} \tau$ is larger than $L_{x}$. As emphasized above, our calculations were performed in the regime in which $l$ is small compared to the strip width. As illustrated in Fig. 3, intersubband transitions contribute to the induced spin-density; electric field induced intersubband coherence cannot be neglected. Although the contribution from each transition is proportional to $\tau$, the number of transitions that are incoherently mixed and contribute is proportional to $\tau^{-1}$, leading to our finding of weak $\tau$ dependence.

We found that in the diffusive region, in which the system size is larger than the mean-free path, the matrix element products that appears in Eq. (5) at $E_{\alpha}-E_{\alpha^{\prime}} \rightarrow 0$ changes slowly with disorder. Exact disorder eigenstates at nearby energies mix 1D band states with disorder free energies that vary over the range $\hbar / \tau$ and in the regime of interest strongly mix states belonging to different subbands. Equation (5) shows that the induced spin-density is proportional to the quantity plotted in the main panel of Fig. 3(a), which varies rapidly with scattering rate when the mean free path exceeds the system size, i.e., low values of $(\hbar / \tau) / E_{F}$, and slowly at larger scattering rates as expected from the above argument. Figure 3(b) shows the eigenenergy difference dependence of the matrix element product which remains large over the energy interval $\Delta_{s o}$.

The above detailed analysis suggests the following physical picutre of the spin Hall accumulation in the strong SO interaction regime. Here we consider a reflection process from a state with a momentum $\mathbf{k}^{\prime}$ to another state $\mathbf{k}$ at the 
left boundary as shown in the Fig. 3(c). In the following we assume the width of stripe $L$ is infinitely large, and focus on the major band. Note that the SO Hamiltonian has a form $H_{s o}=-\boldsymbol{\Delta}(\mathbf{k}) \cdot \boldsymbol{\sigma} / 2$ where the effective Zeeman field is given as $\boldsymbol{\Delta}(\mathbf{k})=2 \lambda k^{\alpha}[-\sin (\alpha \phi), \cos (\alpha \phi), 0]$ with $\alpha=3$ for 2DHGs and $\alpha=1$ for 2DEGs and $\phi=\tan ^{-1}\left(k_{y} / k_{x}\right)$. Thus in a steady state the spins are parallel to the direction of this effective field $\hat{\boldsymbol{\Delta}}=\boldsymbol{\Delta} /|\boldsymbol{\Delta}|$. The dynamics of the direction of spins $\mathbf{m}$ $=\mathbf{S} / S$ is described by the equation of motion

$$
\frac{\partial}{\partial t} \mathbf{m}(\mathbf{k}, t)=\mathbf{m}(\mathbf{k}, t) \frac{\Delta(\mathbf{k})}{\hbar}+\left(\frac{e \mathbf{E}}{\hbar} \cdot \nabla_{\mathbf{k}}\right) \mathbf{m}(\mathbf{k}, t)
$$

in the clean limit. The spin precesses around $\Delta\left(\mathbf{k}^{\prime}\right)$ until the reflection and changes its direction of the precession axis to $\Delta(\mathbf{k})$. In the presence of the electric field $z$ component spins are generated as discussed in the original paper of the intrinsic spin Hall effect. ${ }^{8}$ Assuming $\mathbf{m}(\mathbf{k}, t) \simeq \hat{\mathbf{\Delta}}(\mathbf{k})+\delta \mathbf{m}(\mathbf{k}, t)$, where $\delta \mathbf{m}$ is linear in $e E$, we obtain $(\partial / \partial t)^{2} \delta m_{z}(\mathbf{k}, t)=$ $-|\boldsymbol{\Delta} / \hbar|^{2}\left[\delta m_{z}(\mathbf{k}, t)-m_{z}^{0}(\mathbf{k}, \mathbf{E})\right]$ from Eq. (6). Here note that the static component

$$
m_{z}^{0}(\mathbf{k}, \mathbf{E})=\alpha e \mathbf{E} \cdot[\hat{\mathbf{z}} \times \hat{\mathbf{k}}] / k|\boldsymbol{\Delta}(\mathbf{k})|
$$

causes the spin current density

$$
j^{z}=1 / L^{2} \sum_{\mathbf{k}, M= \pm} f\left(E_{\mathbf{k}, M}\right)\left[M m_{z}^{0}(\mathbf{k}, \mathbf{E}) s \mathbf{k} / m\right]=\sigma_{s H}[\mathbf{E} \times \hat{\mathbf{z}}]
$$

in the bulk, ${ }^{8}$ where $s=3 / 2$ for 2 DHGs. ${ }^{25,26}$ Note that the initial state with $\mathbf{k}^{\prime}$ has a negative $z$ component of spin $m_{z}^{0}$ $=-\alpha e E\left|\hat{k}_{x}^{\prime}\right| / k^{\prime}\left|\boldsymbol{\Delta}\left(\mathbf{k}^{\prime}\right)\right|$, while the right moving reflected state with $\mathbf{k}$ has a positive $m_{z}^{0}=\alpha e E\left|\hat{k}_{x}\right| / k|\boldsymbol{\Delta}(\mathbf{k})|$. A typical amount of $z$ component of spin for all left moving states $\left(k_{x}^{\prime}<0\right)$ is $s / L^{2} \sum_{k_{x}^{\prime} \leqslant 0, k_{y}^{\prime}, M} f\left(E_{\mathbf{k}^{\prime}, M}\right) M m_{z}^{0}\left(\mathbf{k}^{\prime}, \mathbf{E}\right) \simeq-s \alpha e E / 4 \pi v_{F}=-\left|j^{z}\right| / v_{F}$, and the same amount with opposite sign $+\left|j^{z}\right| / v_{F}$ is for all right moving states $\left(k_{x}>0\right)$. Therefore in the bulk of the sample these components cancel each other. On the other hand, near the edge this cancellation cannot be accomplished because it takes a finite time to change the averaged $z$ component spin from $m_{z}^{0}\left(\mathbf{k}^{\prime}, \mathbf{E}\right)$ to $m_{z}^{0}(\mathbf{k}, \mathbf{E})$ by the reflection as indicated schematically in Fig. 3(c). The net spin density arises from this edge induced coherence between different Bloch states and falls to zero on the length scale $2 L_{s o} / \pi$ because of averaging over scattering angles as

$$
S_{z}(x)=-\frac{j_{\text {bulk }}^{z}}{v_{F}} 2 \exp \left(-\frac{\pi}{2} \frac{x}{L_{s o}}\right) .
$$

Here the factor $2 / \pi$ comes from averaging of scattering angles. As shown in Figs. 3(d)-3(f) this ansatz is in a very good agreement with our numerical Kubo formula results. Here we shifted the $x$ coordinate as $x \rightarrow x-x_{\text {edge }}$, where $x_{\text {edge }}$ is the point of the peak of $S_{z}$. These results show a qualitative correspondence between spin accumulation and the bulk SHE with the standard spin-current definition. ${ }^{8}$

We end this theoretical section by noting that qualitatively different behavior is expected for two-dimensional electron systems with Rashba SO coupling. Figure 2(d) shows oscillations of the $z$ component of the 2DEG spin density whose
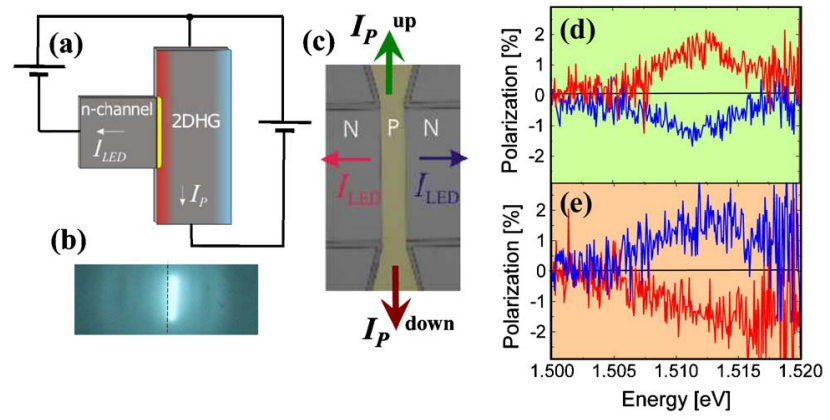

FIG. 4. (Color online) Schematic configuration of lateral $p-n$ junction to detect spin accumulation (a): 2DHG channel bordered by an $n$-type region which forms light-emitting diode (LED). Driving a current $I_{L E D}$ through the LED results in electroluminescence indicated by the yellow line. Driving a current $I_{p}$ along the 2DHG channel induces the SHE spin accumulations at the edges of the 2DHG. Light emission from the $p-n$ junction recorded by a charged-couple device camera (b). Electron microscope image of the microdevice with symmetrically placed LEDs at both edges of the 2DHG channel (c). Emitted light polarization of recombined light in the $p$ - $n$ junction, (d) and (e), for the current flow indicated in (c).

amplitude decreases with increasing system size. The lack of clear edge spin accumulation in this system we obtained numerically is consistent with the suppressed bulk spin current of this model. ${ }^{9-12,17,27}$ A clear edge spin accumulation could appear in the nonlinear transport regime with a strong electric field in a ballistic nanostructre. ${ }^{22}$

\section{EXPERIMENT}

We now show that the above theoretical phenomenology of the SHE in strong SO coupling regime is consistent with our experimental data in modulation doped, GaAs/AlGaAs 2DHG devices. To demonstrate the independence of the SHE on the channel width we compare measurements in the $1.5 \mu \mathrm{m}$ channel $^{4}$ and in a device with a $10 \mu \mathrm{m}$ wide channel, lithographically defined in the same 2DHG system with $l \approx 50 \mathrm{~nm}$. In Fig. 4(a) we show schematically the experimental setup with the LED current, $I_{L E D}$, driving the electroluminescence at the edge of the hole strip, and the 2DHG channel current, $I_{p}$, inducing the SHE edge spin accumulation. Digital camera image of the light emitted from the $p$ $-n$ junction is shown in Fig. 4(b). Figure 4(c) is a scanning electron microscope image of the whole microdevice with symmetrically placed LEDs at both edges of the $10 \mu \mathrm{m}$ channel. Circular polarization of the spectral peak corresponding to recombination of electrons with the spinpolarized 2DHG states at the edges of the channel are shown in Figs. 4(d) and 4(e). For more details on our coplanar LED devices and on the spectroscopical analysis of the electroluminescence data see Refs. 4 and 30 and citations therein.

Consistency of the measured data with the basic SHE phenomenology is demonstrated by the detected opposite sign of the $z$ component of spin accumulation at opposite edges [compare red and blue curves in Figs. 4(d) or 4(e)] and by the observed flipping of the sign of spin polarization at a 
given edge upon reversing $I_{p}$ [compare, e.g., red curves in Figs. 4(d) and 4(e)]. The magnitude of the $I_{p}$ current in the $10 \mu \mathrm{m}$ channel was adjusted so that the corresponding longitudinal electric field, $E_{y} \approx 0.15 \mathrm{~V} \mu \mathrm{m}^{-1}$, was comparable to the field used in the experiment in the $1.5 \mu \mathrm{m}$ channel. ${ }^{4}$ Spin polarizations of order $\sim 1 \%$ observed in both devices confirm the expected independence of the SHE signal on the width of the diffusive channel.

We now compare experimental and calculated magnitudes of the edge spin polarization. Numerical data in Fig. 2 imply an approximate general form of the dimensionless edge polarization

$$
\frac{S_{\text {edge }}^{z}}{\hbar n}=g_{s} \frac{e}{4 \pi v_{F} \hbar n} E_{y},
$$

where $g_{s}$ is a numerical factor. In the measured system the 2 DHG density $n=2 \times 10^{12} \mathrm{~cm}^{-2}, \quad v_{F}=10^{5} \mathrm{~ms}^{-1}, \quad \Delta_{s o} / E_{F}$ $\approx 0.4$, and $(\hbar / \tau) / E_{F} \approx 0.1$. The system is therefore in the strong SO coupling regime and the corresponding numerical factor $g_{s}=7.4$. From Eq. (9) we obtain that the expected polarization for the measured sample reaches $\sim 8 \%$ and the width of the accumulation area is of order $L_{s o} \sim 10 \mathrm{~nm}$. The theoretical $\sim 8 \%$ polarization is consistent with the measured value of $\sim 1 \%$ assuming an effective LED recombination width of the order $\sim 100 \mathrm{~nm}$. This number cannot be precisely determined experimentally because of the resolution limit set up by the wavelength of the emitted light $(\sim 800 \mathrm{~nm})$. However, comparisons between experiments in the $1.5 \mu \mathrm{m}$ and $10 \mu \mathrm{m}$ 2DHG channels, analysis of the digital images of the active $p$ - $n$ junction area, and simulations of device $I-V$ characteristics ${ }^{4}$ confirm a submicron width of the recombination region near the $p-n$ junction.

\section{SUMMARY}

In summary we have studied the edge-spin accumulation due to the SHE in a 2DHG both theoretically and experimentally. We found theoretically a clear correspondence between the edge-spins and the bulk spin currents. The calculated spin-density is nearly independent of the disorder scattering rate in magnitude and is localized within a spin-precession length of the edge. The magnitude increases with the strength of the SO coupling. For the parameters used in the experimental study, we predict an $8 \%$ spin-polarization at the edge. These predictions are consistent with the experimental finding of $\sim 1 \%$ optical polarization averaged over a distance $\sim 10 L_{s o}$. The experiments also confirmed that opposite spin accumulations at the edges can be be separated over large distances in diffusive conduction channels and that their magnitude is not affected by the channel width.

\section{ACKNOWLEDMENTS}

The authors would like to thank S. Murakami, B. A. Nikolic, Q. Niu, S. Onoda, E. Rashba, N. Sugimoto, and S.-C. Zhang for useful discussions. The work has been supported by the Japan Society for the Promotion of Science Grant Agency, by the Welch Foundation, by the U.S. Department of Energy under Grant No. DE-FG03-02ER45958, by the Grant Agency of the Czech Republic through Grant No. 202/ 05/0575, by the Academy of Sciences of the Czech Republic through Institutional Support No. AV0Z10100521, by the Ministry of Education of the Czech Republic Center for Fundamental Research LC510, and by the UK EPSRC through Grant No. GR/S81407/01.
${ }^{1}$ I. Zutic, J. Fabian, and S. Das Sarma, Rev. Mod. Phys. 76, 323 (2004).

${ }^{2}$ C. Day, Phys. Today, 58 (2), 17(2005).

${ }^{3}$ Y. K. Kato, R. C. Myers, A. C. Gossard, and D. D. Awschalom, Science 306, 1910 (2004).

${ }^{4}$ J. Wunderlich, B. Kaestner, J. Sinova, and T. Jungwirth, Phys. Rev. Lett. 94, 047204 (2005).

${ }^{5}$ M. I. Dyakonov and V. I. Perel, Phys. Lett. A, 459 (1971).

${ }^{6}$ J. E. Hirsch, Phys. Rev. Lett. 83, 1834 (1999).

${ }^{7}$ S. Murakami, N. Nagaosa, and S.-C. Zhang, Science 301, 1348 (2003).

${ }^{8}$ J. Sinova, D. Culcer, Q. Niu, N. A. Sinitsyn, T. Jungwirth, and A. H. MacDonald, Phys. Rev. Lett. 92, 126603 (2004).

${ }^{9}$ J. I. Inoue, G. E. W. Bauer, and L. W. Molenkamp, Phys. Rev. B 70, 041303(R) (2004).

${ }^{10}$ E. G. Mishchenko, A. V. Shytov, and B. I. Halperin, Phys. Rev. Lett. 93, 226602 (2004).

${ }^{11}$ R. Raimondi and P. Schwab, Phys. Rev. B 71, 033311 (2005).

${ }^{12}$ Oleg Chalaev and Daniel Loss, Phys. Rev. B 71, 245318 (2004).

${ }^{13}$ E. Rashba, Sov. Phys. Solid State 2, 1109 (1960).

${ }^{14}$ S. Murakami, Adv. Solid State Phys. 45, (2005).

${ }^{15}$ A. G. Mal'shukov and K. A. Chao, Phys. Rev. B 71, 121308(R) (2005).

${ }^{16}$ B. Andrei Bernevig, Taylor L. Hughes, and Shou-Cheng Zhang,
Phys. Rev. Lett. 95, 066601 (2005).

${ }^{17}$ K. Nomura, J. Sinova, N. A. Sinitsyn, and A. H. MacDonald, Phys. Rev. B 72, 165316 (2005).

${ }^{18}$ E. I. Rashba, Phys. Rev. B 68, 241315(R) (2003).

${ }^{19}$ D. Culcer, J. Sinova, N. A. Sinitsyn, T. Jungwirth, A. H. MacDonald, and Q. Niu, Phys. Rev. Lett. 93, 046602 (2004).

${ }^{20}$ P. Zhang, J. Shi, D. Xiao, and Q. Niu, cond-mat/0503505 (unpublished).

${ }^{21}$ X. Ma, L. Hu, R. Tao, and S.-Q. Shen, Phys. Rev. B 70, 195343 (2004).

${ }^{22}$ B. K. Nikolić, S. Souma, L. P. Zârbo, and J. Sinova, Phys. Rev. Lett. 95, 046601 (2004).

${ }^{23}$ Q. Wang, L. Sheng, and C. S. Ting, cond-mat/0505576 (unpublished).

${ }^{24}$ M. Onoda and N. Nagaosa, cond-mat/0505437 (unpublished).

${ }^{25}$ R. Winkler, Phys. Rev. B 62, 4245 (2000).

${ }^{26}$ J. Schliemann and D. Loss, Phys. Rev. B 71, 085308 (2005).

${ }^{27}$ A. Mal'shukov, L. Wang, C. Chu, and K. Chao, cond-mat/ 0506724 (unpublished).

${ }^{28}$ M. Governale and U. Zülicke, Phys. Rev. B 66, 073311 (2002).

${ }^{29}$ Gonzalo Usaj and C. A. Balseiro, Europhys. Lett. 72, 631 (2005).

${ }^{30}$ B. Kaestner, J. Wunderlich, J. Sinova, and T. Jungwirth, condmat/0507143 (unpublished). 